\title{
Posterior Cruciate Ligament Ganglion: Case Report
}

\author{
Kosei Ishigaki, Hideyuki Aoki*, Yoshiyasu Miyazaki, Takashi Saito, Taisei Sako, \\ Ayaka Kuzuhara, Kazuaki Tsuchiya, Takashi Nakamura \\ Toho University, Tokyo, Japan \\ Email: *aokin8aokin8@gmail.com
}

Received 19 March 2016; accepted 7 May 2016; published 10 May 2016

Copyright (C) 2016 by authors and Scientific Research Publishing Inc.

This work is licensed under the Creative Commons Attribution International License (CC BY). http://creativecommons.org/licenses/by/4.0/

cC) (7) Open Access

\begin{abstract}
Intra-articular ganglion of the knee is uncommon and it shows non-specific symptoms. In this report, we present for a treatment of the ganglion arising from the PCL and the mechanism of pain. The case is a 33-year-old male with pain in his left knee. On presentation, he had no history of locking or giving way of the knee, but had pain on standing. At the first medical examination, the range of motion of his knee was full, with no loss of extension, and there was no effusion or swelling, but the terminal knee flexion was painful. McMurray's test was negative, and the collateral and cruciate ligaments were clinically stable. On MRI, ganglion cysts droved from PCL appear as well-defined cystic multiloculated masses. The patient underwent arthroscopy through the standard anteromedial and anterolateral portals. Consequently, it was diagnosed as a ganglion cyst. The pain disappeared completely after the surgery and neither PCL ganglion nor symptoms recurred up to 9 months post-surgery. The intra-articular ganglion of the knee often is asymptomatic and discovered incidentally. But the reports of ganglion cysts of the knee have been increasing with the increasing availability and use of MRI. We considered that the mechanism of the pain due to ganglion in the cruciate ligament was stimulation of the synovial nerve terminal. One key point for consideration in this study is that arthroscopy should be performed through posterior portal to observe the whole of the PCL and ganglion cysts because it was difficult to observe both the PCL and ganglion cysts with anterior portal. While arthroscopy did not confirm continuity of the ACL and meniscus, it was considered that the ganglion cysts developed in the PCL because of the continuity with the PCL.
\end{abstract}

\section{Keywords}

PCL, Posterior Cruciate Ligament, Ganglion

\footnotetext{
${ }^{*}$ Corresponding author.
}

How to cite this paper: Ishigaki, K., Aoki, H., Miyazaki, Y., Saito, T., Sako, T., Kuzuhara, A., Tsuchiya, K. and Nakamura, T. (2016) Posterior Cruciate Ligament Ganglion: Case Report. Open Journal of Orthopedics, 6, 109-112. 


\section{Introduction}

A ganglion is a small multilocular cyst that can often develop on the wrist and back of the hand. Cann et al. reported the development of a ganglion in the joint for the first time in 1924 [1]. Krudwing et al. showed that most of intra-articular ganglions are developed in the anterior cruciate ligament (ACL) and posterior cruciate ligament (PCL) [2]. With increasing the use of magnetic resonance imaging (MRI), the numbers of ganglion cystreports were increased. Intra-articular ganglion of the knee is uncommon and it shows non-specific symptoms. In this report, we present for a treatment of the ganglion arising from the PCL and the mechanism of pain.

\section{Case Report}

A 33-year-old male with pain in his left knee. He had noticed the pain after playing football two years ago, however the symptoms were reduced on the following day, he continued with his regular activities. Later the symptom became worse, so he came to our hospital. On presentation, he had no history of locking or giving way of the knee, but had pain on standing. At the first medical examination, the range of motion of his knee was full, with no loss of extension, and there was no effusion or swelling, but the terminal knee flexion was painful. McMurray's test was negative, and the collateral and cruciate ligaments were clinically stable. The patellofemoral joint surface was normal, and patellar movements were normal.

Plain radiograph showed no abnormal finding. On MRI, ganglion cysts droved from PCL appear as well-defined cystic multiloculated masses (Figures 1(a)-(c)). They have uniformed low signal intensity on T1-weighted imaging and high signal intensity on T2-weighted imaging.

The patient underwent arthroscopy through the standard anteromedial and anterolateral portals (Figure 2(a) and Figure 2(b)). The ganglion cysts were located between ACL and PCL, arising from the anterior surface of PCL. There was no continuously with ACL and meniscus. It encased in fibrous tissue and synovium and easily punctured by the forceps. A slightly yellow colored viscous fluid was leaked from the cysts. The wall of the cyst was resected piece by piece with the forceps and shaver until the front of the PCL was observed.

Histological examination shows that cystic lesion consists of dense fibrous connective tissue without synovial lining cells (Figure 3). Consequently, it was diagnosed as a ganglion cyst.

The pain disappeared completely after the surgery and neither PCL ganglion nor symptoms recurred up to 9 months post-surgery.

\section{Discussion}

A ganglion is a small multilocular cyst that can often develop on the wrist and back of the hand.The intra-articular ganglion of the knee often is asymptomatic and discovered incidentally [3]. Pathogenetic mechanism remains unclear and possible causes include synovial protrusion, degeneration of connective tissues due to

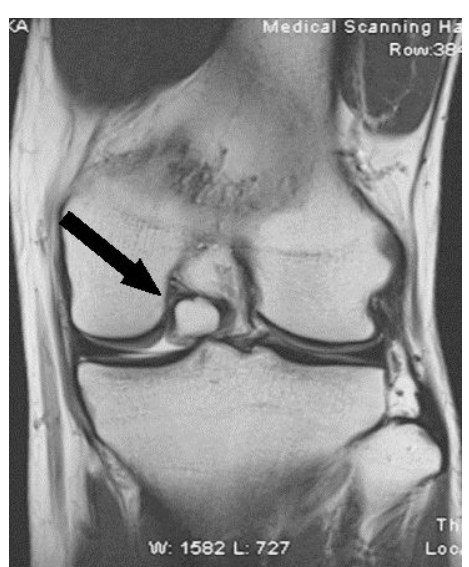

(a)

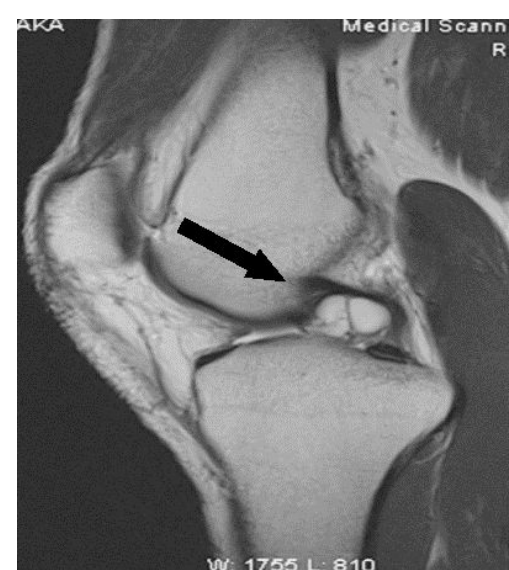

(b)

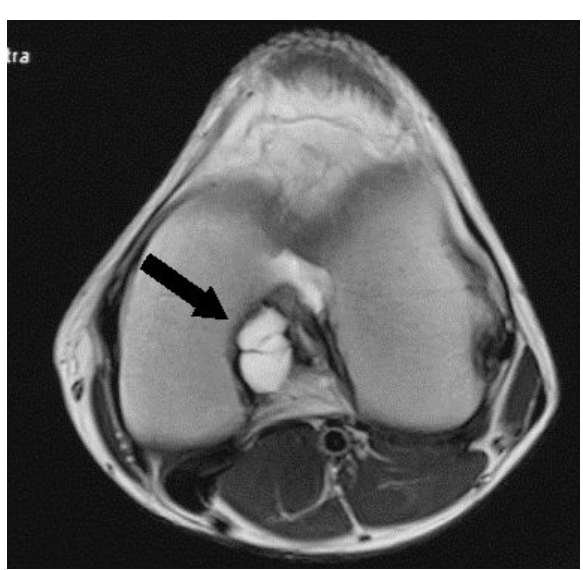

(c)

Figure 1. Sagittal proton-density-weighted (a), coronal (b), and axial (c) magnetic resonance imaging of left knee shows area of high signal intensity. This signal abnormality represents a multilobulated ganglion cyst (arrows) of the posterior cruciate ligament. 


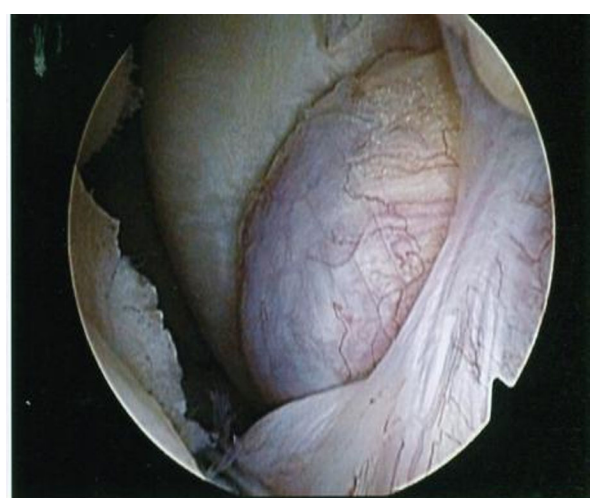

(a)

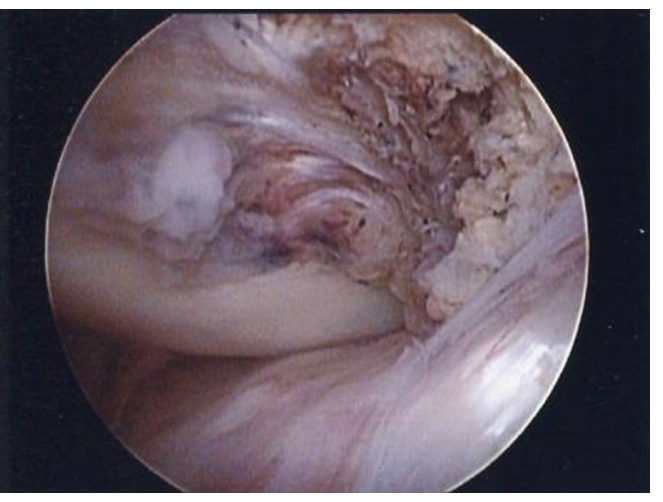

(b)

Figure 2. Arthroscopy shows a cyst (large arrow) between the anterior cruciate ligament and the medial femoral condyle (small arrow) in the left knee.

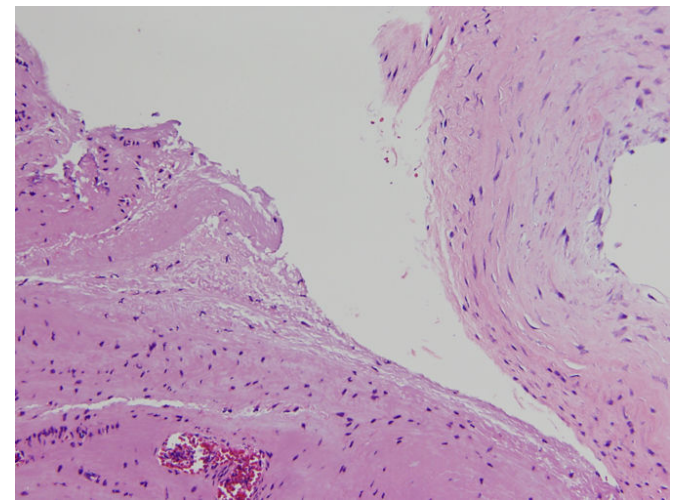

Figure 3. Cystic lesion consists of dense fibrous connective tissue without synovial lining cells (Hematoxylin and eosin 200×).

trauma, degeneration of connective tissues to mucous component, ectopic changes in the synovium, true tumor and persistent fetal synovium. Brown et al. found ganglion in 38 (0.58\%) of 6500 patients who underwent arthroscopy and only $6(0.09 \%)$ of them were arising from PCL [4]. Krudwing et al. showed that intra-articular ganglion were found in 85 (1.06\%) of 8000 patients and while many of them arising from the cruciate ligament, most were asymptomatic [2]. Case reports of ganglion cysts of the knee have been increasing with the increasing availability and use of MRI. However, ganglion cysts arising from the PCL are still rare.

Maeno et al. classified the site of ganglion in the cruciate ligament into three categories, Group I: anterior to the ACL, Group II: between the ACL and PCL, and Group III: posterior to the PCL [5]. They proposed that decreased ROM was secondary to a mechanical block caused by the ganglion cyst. Our case, the ganglion was located between the ACL and PCL; therefore, it was classified into Group II. The symptom of this case was pain when bending, which is similar to the flexion disorder that is often found in Group II. It has also been proposed that limited ROM is actually caused by stimulation of nerve endings in the synovial membrane during cyst compression. Nociceptors are distributed in the joint capsules, ligaments and fat bodies. Hirasawa et al. [6] showed that many nociceptors were detected in the joint capsules and synovia around the sites attached to the meniscus and surrounding attachment sites with the central and peripheral ligaments. Nociceptors in the joint respond to changes in internal pressure, nonphysiological stress, physical stimulation and inflammatory products, resulting in pain. We considered that the mechanism of the pain due to ganglion in the cruciate ligament was stimulation of the synovial nerve terminal.

There is no consensus on the ideal treatment algorithm for intra-articular ganglions. If there is no symptom, we should wait and watch to make sure that no unusual changes occur. However, if it causes pain because of pressuring on the nerves or ligaments, it should be aspiration by needle or resection under arthroscopy. It is reported that arthroscopic resection is the most common and preferred approach because there is a very low rate of 
recurrence, on the other hand, there is possibility of reappearing after aspiration [2] [7].

One key point for consideration in this study is that arthroscopy should be performed through posterior portal to observe the whole of the PCL and ganglion cysts because it was difficult to observe both the PCL and ganglion cysts with anterior portal. While arthroscopy did not confirm continuity of the ACL and meniscus, it was considered that the ganglion cysts developed in the PCL because of the continuity with the PCL.

\section{Conclusion}

A ganglion arising from PCL is rare. Nonphysiological stress to the ACL or PCL that was protruded by the ganglion causes the pain. We recommend arthroscopy for removing intra-articular ganglions.

\section{Consent}

The patient gave informed consent to submit this case study for publication.

\section{Conflicts of Interest}

The authors declare that they have no conflict of interest.

\section{References}

[1] Cann, P. (1924) Zystenbildung (Ganglion) imligamentumcruciatum ant genus. Deut Z Chir, 186, 403-408.

[2] Krudwig, W.K., Schulte, K.K. and Heinemann, C. (2004) Intra-Articular Ganglion Cysts of the Knee Joint: A Report of 85 Cases and Review of the Literature. Knee Surgery, Sports Traumatology, Arthroscopy, 12, 123-129. http://dx.doi.org/10.1007/s00167-003-0372-9

[3] Shetty, G.M., Nha, K.W., Patil, S.P., et al. (2008) Ganglion Cysts of the Posterior Cruciate Ligament. The Knee, 15, 325-329. http://dx.doi.org/10.1016/j.knee.2008.02.010

[4] Brown, M.F. and Dandy, D.J. (1990) Intra-Articular Ganglia in the Knee. Arthroscopy, 6, 322-323. http://dx.doi.org/10.1016/0749-8063(90)90064-K

[5] Maeno, S., Suda, Y., Fukui, Y., et al. (2006) Causes of Limitation of Knee Range of Motion in the Patients with Cruciate Ganglion. Arthroscopy, 31, 177-181.

[6] Hirasawa, T. (2003) Pain Factor in Osteoarthritis of the Knee. Meiji Shinkyu Igaku, 32, 1-8. (In Japanese)

[7] DeFriend, D.E., Schranz, P.J. and Silver, D.T. (2001) Ultrasound Guided Aspiration of the Posterior Cruciate Ganglion Cysts. Skeletal Radiology, 30, 411-414. http://dx.doi.org/10.1007/s002560100374 